Deutsch lernen nach (und trotz) der Bildungsreform in Frankreich - eine Zwischenbilanz

\title{
Laure Gautherot
}

\section{(2) OpenEdition Journals}

Édition électronique

URL : http://journals.openedition.org/esp/1578

DOI : 10.4000/esp. 1578

ISSN : 2532-0319

Éditeur

Centre d'Information sur l'Éducation Bilingue et Plurilingue

\section{Édition imprimée}

Date de publication : 1 décembre 2017

Pagination : 65-75

ISSN : 1127-266X

\section{Référence électronique}

Laure Gautherot, « Deutsch lernen nach (und trotz) der Bildungsreform in Frankreich - eine

Zwischenbilanz », Éducation et sociétés plurilingues [Online], 43 | 2017, Online erschienen am: 01

Februar 2019, besucht am 30 April 2019. URL : http://journals.openedition.org/esp/1578; DOI

10.4000/esp.1578 


\section{DEUTSCH LERNEN NACH (UND TROTZ) DER BILDUNGSREFORM IN FRANKREICH - EINE ZWISGHENBILANZ}

\section{Laure GAUTHEROT}

Cet article propose de revenir sur les conséquences de la réforme du collège de 2015 pour l'apprentissage de l'allemand et les conditions de sa mise en place à la rentrée 2016/2017. La réflexion sur l'arrêté du 16 juin 2017 publié par le nouveau gouvernement permet de mesurer si les modifications apportées relèvent d'un rétropédalage institutionnel ou d'une possibilité de restaurer ce qui a été détruit par la réforme de la précédente ministre de l'Éducation Nationale. La dernière partie examinera diverses actions inscrites dans une constante de la politique éducative de ces quinze dernières années: faciliter l'intégration des apprenant.e.s dans le monde professionnel par le développement des compétences.

Mots-clés: apprendre l'allemand, France, réforme du collège 2015, arrêté du 16-06-2017, Ministère de l'Éducation Nationale.

This article focuses on the consequences of the secondary school reform in France for learning German in 2015 and its implementation in 2016/2017. The by-law decided by the new government on June 16, 2017 allows us to judge whether the latest changes are due to retrograde politics or to the possibility to restore the measures the previous reform destroyed. Several concrete actions are examined as being regular and recurrent features of the French education system over the last fifteen years: facilitating the integration of students into the business world by skills development.

Keyzeords: Learning German in France, Secondary school reform 2015, bylaw of 16-06-2017, French System of Education.

Quest'articolo propone di tornare sulle conseguenze della riforma della scuola media del 2015 per l'apprendimento del tedesco in Francia e sulle condizioni della sua applicazione durante l'anno scolastico 2016/2017. La riflessione sull'ordinanza del 16 giugno 2017 pubblicata dal nuovo governo permette di valutare se le modifiche apportate siano da considerare un freno posto delle istituzioni oppure una possibilità di restaurare quello che è stato distrutto dalla precedente ministra dell'Istruzione. L'ultima parte esaminerà varie azioni iscritte in una costante della politica educativa degli ultimi quindici anni: facilitare l'integrazione degli apprendenti nel mondo professionale grazie allo sviluppo delle competenze.

Parole chiave: apprendimento del tedesco, Francia, riforma della scuola media 2015, ordinanza del 16-06-2017, Ministero Istruzione francese.

KONTEXTUALISIERUNG as letzte Schuljahr 2016/2017 in Frankreich hat in einem gespannten Kontext mit der Einführung der umstrittenen Schulreform der damaligen Bildungsministerin Najat Vallaud-Belkacem in den Collèges (gymnasialen Mittelstufen) begon- 
Deutsch lernen nach (und trotz) der Bildungsreform in Frankreich - eine Zwischenbilanz

L. GAUTHEROT nen, was die deutsche Botschafterin in Paris, Susanne WasumRainer, schon im April 2015 dazu gebracht hatte, vor der „Gefahr einer atmosphärischen Beeinträchtigung unserer bilateralen Abkommen und Absprachen" (Die Welt 23.04.2015 [Online]) zu warnen. Sorgen haben sich seitdem in den französischen und deutschen Medien verbreitet, Petitionen, offene Briefe an die Bildungsministerin und an französische Abgeordnete, Informationsveranstaltungen (wie der Informationsabend des Goethe Instituts am 6. Mai 2015 in Paris) bis zu LehrerInnenstreiks haben vom Frühling 2015 bis zum Frühling 2017 in ganz Frankreich stattgefunden. Persönliche Stellungnahmen von französischen Politikern gegen die Reform, auch aus der eigenen Partei der Ministerin, hatten sich vermehrt: Ex-Premierminister Jean-Marc Ayrault, der ehemalige Kulturminister Jacques Lang und der Abgeordnete der Franzosen im Ausland Pierre-Yves Le Borgn haben ihre Sorge um die Zukunft der französischen deutschsprachigen SchülerInnen und der deutsch-französischen Beziehungen wiederholt offiziell geäußert. In seinem Brief vom 25.03.2015 an die Bildungsministerin teilt Le Borgn sein Unverständnis der Reform und vor allem seine große Sorge um die Zukunft der deutschen Sprache in Frankreich mit. Er bittet auch die Bildungsministerin um ein offizielles Gespräch mit Thérèse Clerc, Präsidentin des Deutschlehrerverbandes $\Lambda \mathrm{DE} A \mathrm{~F}$ (Association pour le Développement de l'Enseignement de l'Allemand en France), das niemals gewährt wurde.

Die großen Wellen, die die Bildungsreform in Bezug auf den Deutschunterricht geschlagen hat, lassen sich dadurch erklären, dass sie zwei Meilensteine der deutsch-französischen Geschichte und Zusammenarbeit verletzt. Zuerst wird die Abschaffung eines intensiven Deutschunterrichts als Beleidigung des historischen, hoch symbolischen Elysée-Vertrags angesehen. Dann verstößt der Angriff auf den Deutschunterricht in Frankreich gegen die gemeinsame Verpflichtung der Regierungen in den deutsch-französischen Ministerräten 2003 in Paris und 2013 in Berlin (jeweils zum 40. und 50. Jahrestag des ElyséeVertrages), strukturelle Maßnahmen zur Förderung des Deutschunterrichts in Frankreich und des Französischunterrichts in Deutschland zu ergreifen.

Inzwischen wurde die Bildungsreform in den Präsidentschaftswahlen 2017 politisch aufgeladen und in den Programmen der wichtigsten Kandidaten als Wahlkampfthema aufgegriffen. Vom Sieg des jungen parteilosen Emmanuel Macron haben sich viele Germanisten und DeutschlehrerInnen eine spätere Aufhebung der Bildungsreform erhofft, denn er hatte sich, ähnlich wie seine Kontrahenten François Fillon und Jean-Luc Mélenchon, für dic Wiedereinrichtung der bilin- 
Deutsch lernen nach (und trotz) der Bildungsreform in Frankreich - eine Zwischenbilanz

L. GAUTHEROT

DIE BILDUNGSREFORM UND DER DAFUNTERRICHT 2016 IM COLLÈGE gualen Klassen in allen französischen Regionen ausgesprochen. Wie sich die Einführung der Bildungsreform in den Collèges auf den Deutschunterricht ausgewirkt hat und wie die Situation nach Regierungswechsel nun aussieht, wird in diesem Artikel untersucht.

Die französische Schulreform des Jahres 2015/2016 betrifft in erster Linie den DaF-Unterricht im Collège mit der programmierten Abschaffung der bilingualen Klassen (classes bilangues) und der Europa-Klassen (sections européennes). Das System der bilingualen Klassen kann wie folgt skizziert werden: Ab der sechsten Klasse (6ème) wird neben Englisch eine zweite Fremdsprache (in der Regel drei Stunden pro Woche) unterrichtet, was den Schülerinnen und Schülern ermöglicht, ab der sechsten Klasse zwei Fremdsprachen gleichzeitig zu lernen, wobei Deutsch mehrheitlich gewählt wird. Die bilingualen Klassen wurden einstimmig als Stütze für den DaFUnterricht in Frankreich betrachtet: ab 2005 sei eine Stabilisierung um die 15\% Deutschlernenden zu vermerken (Hannequart 2013:1, zitiert nach Ammon, 2015: 996). Aber aufgrund ihres „elitären Charakters" erhalten sic laut Bildungsministerin dic sozialen Ungleichheiten aufrecht. (1) Deshalb wurden sie durch die Reform gestrichen und durch die allgemeine Einführung der zweiten Fremdsprache in der 7. Klasse für alle Schüler/innen (ein Jahr früher als bis dahin) ersetzt. Inzwischen wurde die Entscheidung der Bildungsministerin teilweise revidiert und seit dem 22. Januar 2016 - Tag der deutsch-französischen Freundschaft - öffentlich betont: wenn mit dem Erlernen einer anderen Fremdsprache als Englisch in der Primarstufe angefangen wurde, könnte der Unterricht zweier Fremdsprachen in der 6. Klasse theoretisch fortgeführt werden. Es sind die sogenannten ,bilangues de continuité" (bilinguale Fortführungsklassen). Dennoch stellten sich zwei Probleme:

ein Interpretationsproblem, denn die Bedingungen zum Übergang der bilingualen Klassen in Fortführungsklassen wurden von den schulischen Aufsichtsinstitutionen (Rectorats) und Schulleitungen in manchen Regionen nicht gleich interpretiert. Deutschlehrer/innen berichten von Ablehnungen durch die entsprechenden Instanzen, trotz erfüllter Bedingungen (ausreichender Anzahl an eingeschriebenen Schülern und DaF-Unterricht in der Primarstufe), im Schuljahr 2016/2017 eine bilinguale Klasse in Fortführungsklasse aufrechtzuerhalten (ADEAF 2016 [Online]).

ein Problem der Stundenzahl, denn die zweite Fremdsprache wurde in den Fortführungsklassen nur noch zweieinhalb und nicht mehr drei Stunden pro Woche unterrichtet, was vor allem in den ersten Lehrjahren für den Spracherwerb bedeutsam ist. 
Deutsch lernen nach (und trotz) der Bildungsreform in Frankreich - eine Zwischenbilanz

L. GAUTHEROT

DIE SCHLACHT UM DIE ZAHLEN

\section{DIE}

AKTUALISIERUNG

DER REFORM

NACH DEM

REGIERUNGSWEGHS

EL 2017
Bei den ersten Ergebnissen der Reform des Deutschunterrichts fällt die Schlacht um die Zahlen zwischen Bildungsministerium und Deutschlehrerverband ADEAF auf. Laut Ersterem sei im September 2016 ein Anstieg von 6\% der Deutschlernenden im Collège zu vermerken (2), während der Verband von einem Rückgang von 26\% in der 6. Klasse spricht

(http://adeaf.net/IMG/pdf/politique_des_langues_et_allemand-_contribution_de_1_adeaf_2017.pdf).

Der Anstieg des Bildungsministeriums lässt sich dadurch erklären, dass in dem Einführungsjahr der Reform zwei Systeme parallel verlaufen. Dieses Jahr - und nur dieses eine Jahr - beginnen alle französischen Schüler/innen der 7. und der 8. Klasse mit dem Erlernen einer zweiten Fremdsprache. Mehr Schüler denn je lernen also de facto zwei Fremdsprachen im Collège, darunter auch Deutsch. Daher können diese Zahlen keine Indikatoren für die Entwicklung der Deutschlernenden in den kommenden Jahren sein und dienen durch ihre argumentative Funktion dem „,Ethos der Kompetenz der Ministerin“ (Durand 2017: 194).

Im Unterschied dazu verweist die ADEAF auf das Ergebnis einer Felduntersuchung, in dem ein Rückgang von $26 \%$ der Deutschlernenden in der 6. Klasse im September 2016 als Folge der Schließung von 1/3 der bilingualen Klassen festgestellt wird. Vor allem in den Pariser Vororten und den grenznahen Regionen Elsass und Lothringen bestehen die bilingualen Klassen weiter, jedoch nicht ohne Klassenschließungen. In einer Umfrage zum Schuljahr 2016/2017 bei den Schulleiter/innen der Straßburger Académie werden die bilingualen Klassen der 43 Collèges, die an der Umfrage teilgenommen haben, in 42 beibehalten. Von den 7 Collèges, die bisher von der 6. bis zur 9. Klasse ausschließlich bilinguale Klassen anboten, verfugen nur noch 5 weiterhin über ein solches Angebot (Geiger-Jaillet, Rothacker 2017: 87).

Nach den Präsidentschaftswahlen im Mai 2017 in Frankreich herrschten zuerst Euphorie nach dem Sieg des proeuropäischen Kandidaten und eine gewisse Erleichterung darüber, dass die Kandidatin der rechtsextremistischen Partei Front National nicht gewählt worden war. Der neue Präsident Macron schien sich von Anfang an der Tatsache bewusst zu sein, dass die deutsch-französischen Beziehungen auf gegenseitigem Verständnis und dementsprechend auf dem Erlernen der Nachbarsprache beruhen. Der Antrittsbesuch (knapp einen Tag nach Amtseinführung) in Berlin und die Ernennung der Deutschlandkenner Bruno Lemaire zum Wirtschaftsminister und Sylvie Goulard zur Verteidigungsministerin konnten Germanisten zuerst einmal freudig stimmen und als deutschfreundliches, positives Signal gedeutet werden. 
Deutsch lernen nach (und trotz) der Bildungsreform in Frankreich - eine Zwischenbilanz

L. GAUTHEROT
DIE ,EPIS“,

ÜBERBLEIBSEL DER REFORM FÜR DIE NEUERUNG DES DAF-UNTERRICHTS
Beide Politiker hatten sich nämlich 2015 eindeutig und vehement gegen die Bildungsreform und die Abschaffung der bilingualen Klassen Bruno Le Maire in einem Fernseh-Interview mit Najat VallaudBelkacem und Sylvie Goulard unter anderem beim Informationsabend des Goethe Instituts in Paris - ausgesprochen. Das Treffen zwischen Mitarbeitern des neuen Bildungsministers Jean-Michel Blanquer und einer Delegation des Deutschlehrerverbands ADEAF am 7. Juli 2017 zeugte auch von einer offensichtlich gutwilligen Haltung der höheren Instanzen und lässt womöglich auf künftige Kooperation statt bisherigen Ignorierens hoffen. Ob diese demonstrativen Zeichen nur ausgewählte Stücke einer neuen Kommunikationsstrategie bilden, wird sich erst in der Zukunft prüfen lassen.

Nichtsdestotrotz wurde auf juristischer Ebene die Bildungsreform der vorherigen Regierung nicht abgeschafft, sondern mit dem Erlass vom 16. Juni 2017 lediglich gelockert und in einigen Punkten revidiert. Den Schulleitungen wird in den Artikeln 2 und 4 vor allem eine größere Unabhängigkeit und Organisationsfreiheit gewährt. Die Wiederherstellung der bilingualen Klassen und der Europa-Klassen steht zwar nicht schwarz auf weiß, dennoch wird sie anhand der Artikel 3 und 5 im Rahmen des dreistündigen optionalen Unterrichts ermöglicht. Die Bedingungen zum Erhalt der bilingualen Klassen in der Form von Fortführungsklassen werden durch Artikel 5b de facto aufgehoben, indem eine zweite Fremdsprache ab der 6. Klasse unterrichtet werden darf (solange der gesamte Fremdsprachenunterricht der beiden Sprachen nicht 6 Stunden überschreitet). Ein zweistündiger Unterricht der „europäischen Sprachen und Kulturen“ (in Erweiterung des Fremdsprachenunterrichts) wird im Artikel $5 \mathrm{c}$ ab der 7. Klasse erlaubt, was die Europa-Klassen wieder ins Leben ruft. Jedoch beträgt die übliche Stundenzahl der zweiten Fremdsprache ab der 5. Klasse wie nach der Reform zweieinhalb Stunden pro Woche. Um die gleiche Stundenzahl wie in den ehemaligen bilingualen Klassen zu erreichen, wird zwar theoretisch ein Zusatz von 0,5 Stunden durch Aufteilung des optionalen zweistündigen Unterrichts der „,europäischen Sprachen und Kulturen" ermöglicht, aber diese steht unter Zustimmungsvorbehalt des Verwaltungsrats des jeweiligen Collège.

Von der Bildungsreform ist nach Regierungswechsel der Vorschlag der „EPIs“ (Enseignements Pratiques Interdisciplinaires) beibehalten worden. Auch wenn das Wort - ähnlich wie für die bilingualen und Europa-Klassen - im Erlass nicht mehr vorkommt, zählt die fächerübergreifende Unterrichtsform im Artikel 5 zum möglichen optionalen Unterricht ab der 7. Klasse. Jeder und jede französische Schüler/in kann von einer bis drei Stunden pro Woche Unterricht 
Deutsch lernen nach (und trotz) der Bildungsreform in Frankreich - eine Zwischenbilanz

L. GAUTHEROT
EDITORISCHE DIDAKTISIERUNGSV ORSCHLÄGE in der Form eines optionalen Unterrichts bekommen' dessen pädagogischer Inhalt sich von zwei Themen ableitet: Sprachen und Kulturen der Antike; Regional- oder Fremdsprachen und Kulturen. In einem praktischen Ratgeber hatten die Inspektoren der Straßburger Académie darauf hingewiesen, dass die Fremdsprachen und die neuen Technologien in den EPIs besonders zum Einsatz kommen sollten. (3) Von den neunzehn konkreten Beispielen auf der Website der Académie nehmen drei explizit Bezug auf Deutsch (,Trier gestern und heute“; „Berlin“; ,Ein gesundes Frühstück" "), eines auf Italienisch und drei auf Fremdsprachen im Allgemeinen. Jedes Mal geht es darum, deutsche Kultur zu vermitteln und die deutsche Sprache in die Realisierung des Projekts einzubinden. Dennoch wurde diesen EPIs die geringe Anwendbarkeit der deutschen Sprache in den Projekten häufig vorgeworfen, vor allem in den frühen Klassen (7. und 8. Klasse), in denen die unzureichenden Fremdsprachenkenntnisse und Kompetenzen der DaF-Lernenden die Nutzung der Fremdsprache begrenzen. Aus diesem Grund wurden die EPIs als ,ministerielles Aushängeschild der Reform“ kritisiert. Im politischen Wochenmagazin Le Point wurde zum Beispiel über die EPIs zum Artikel ein Begleitfoto veröffentlicht, das folgende Anspielung veranschaulicht: Im Rahmen eines EPIs um Harry Potter hatte sich die Bildungsministerin verkleidet und mitgespielt. Der polemische Titel weist auf das leichtsinnige, potentiell gefährliche Experiment der unerfahrenen Bildungsministerin hin, indem sie als ,Zauberlehrling" präsentiert wird (Coignard 2017 [Online]).

Ein weiterer Vorwurf, der den EPIs der Reform und dem optionalen Unterricht des letzten Erlasses gilt, betrifft die schwer zu organisierende Verteilung und Einbettung der betroffenen Fächer in den Stundenplan. In diesem Fall stößt die fächerübergreifende Absicht des Unterrichts erstens auf eine strukturelle Komponente des französischen Staatsexamens zum Lehramt, nämlich die monovalente Ausbildung der LehrerInnen der Mittel- und Oberstufe des französischen Gymnasiums. (4) Für eine fächerübergreifende effektive Zusammenarbeit in den Lehrerkollegien erscheint daher eine umfangreiche Fortbildung derselben Lehrer/innen erforderlich. Zweitens wird der größere Spielraum der Schulen in der Wahl des optionalen Unterrichts zu wichtigen Unterschieden von einer Schule zur anderen beitragen und eventuell die Konkurrenz zwischen den verschiedenen Fächern und Lehrerkollegien einer und derselben Schule anspornen.

Um das Hindernis der geringen Fremdsprachenkenntnisse in den ersten Lehrjahren zu umgehen, schlagen die letzten Handbücher der französischen Verlage EMDL (Editions Maison des Langues) und Hatier seit 2016 mehrere EPIs vor. Zum Beispiel bietet das 
Deutsch lernen nach (und trotz) der Bildungsreform in Frankreich - eine Zwischenbilanz

L. GAUTHEROT

\section{SCHWERPUNKT \\ AUF DEM ÖKONOMISCHEN INTERESSE}

Handbuch Fantastisch drei EPIs für das erste Lehrjahr: „Une année en fête" (Ein Festjahr); „Danser les mots" (mit Wörtern tanzen); „Animaux fantastiques“ (fantastische Tiere), bei denen Deutsch immer im Zusammenhang mit Musikunterricht und dazu Sport oder Kunst gebraucht wird (Fantastisch! 2016: 125ff.). Somit wird der Mangel an Deutschkenntnissen durch kreative Aktivitäten überwunden - oder, um es anders auszudrücken, verdeckt. Ab dem zweiten Lehrjahr werden 2 EPIs vorgeschlagen, in denen die mehrsprachigen Kompetenzen der Schüler/innen in Deutsch und Englisch (eventuell auch Italienisch und Spanisch) deutlich mehr eingesetzt werden, wie zum Beispiel: „Une langue, une vie“(Eine Sprache, ein Leben) und „Il était une fois... le bonheur" (Es war einmal... das Glück) im dritten Lehrjahr (Fantastisch! 3ème année 2017: 116-117). Der Verlag Hatier setzt auf eine digitale Strategie und stellt die Vorschläge seiner Reihe Blick und Klick nicht in den Handbüchern (bzw. in den sogenannten „LernBüchern"), sondern in einer allgemeinen online Datenbank zur Verfügung. Damit sollen die Hilfsmittel und das Projekt der kollektiven Gestaltung der verschiedenen Lehrer/innen angepasst werden.

Die wohl einzige Motivation zum Erlernen der deutschen Sprache, worüber Konsens herrscht und die von den deutschen LehrerInnen bei ihren Werbetouren in den Grundschulen gerne als Vorteil präsentiert wird, ist das ökonomische Interesse für Deutschlernende, denn der deutsche Arbeitsmarkt bleibt für Franzosen (insbesondere für Pendler der Nachbarregionen) attraktiv. In dieser Hinsicht versucht der DaF-Unterricht in Frankreich, die Lernenden auf die Arbeitswelt besser vorzubereiten. Dieser Kurswechsel der französischen Bildungspolitik in Richtung übertragbare Kommunikationsstrategien zeugt von einer immer größeren Anpassung an die Forderungen der modernen Arbeitswelt. Daher erscheinen das bildungserziehende Prinzip der Bewertung und Unterrichtsgestaltung nach den Kompetenzen der Lernenden und die Forderung der polarisierten Arbeitswelt sehr eng miteinander verbunden. Insofern hat sich das 2002 schon von Schneider-Mizony „neue Erziehungsparadigma, dass die Schule nicht dazu da sei, den Menschen zu bilden, sondern ihn aufs Berufsleben vorzubereiten" (Schneider-Mizony 2002: 23) heute umso mehr bekräftigt. Anstatt kulturelle und sprachliche Kenntnisse zu erwerben wird zum effizientesten Gebrauch von Kommunikationsstrategien in bestimmten Kontexten trainiert. Mit weniger als drei Stunden Unterricht pro Woche in der ganzen gymnasialen Oberstufe wird jedoch das dazu nötige Sprachniveau wie auch eine interkulturelle Kompetenz schwer 
Deutsch lernen nach (und trotz) der Bildungsreform in Frankreich - eine Zwischenbilanz

L. GAUTHEROT
ZWISCHENBILANZ UND AUSSICHTEN zu erwerben sein.

In diesem Sinne bemühen sich die deutsche und die französische Regierungen um eine enge Kooperation in grenznahen Gegenden, indem sie mehrere Vereinbarungen zur Mobilität der jungen Lernenden in einer beruflichen Perspektive getroffen haben. In der Erklärung von Saarbrïcken vom 15. Juli 2013 zwischen Beauftragten für die deutsch-französische Zusammenarbeit der grenznahen Regionen Saarland / Lothringen und Elsass / Ortenau stehen auf Punkt 1 und Punkt 2 der Richtlinien „Bildung und Ausbildung“ und „Arbeitsmarkt", vor den Punkten „Polizeiliche Zusammenarbeit“, „Grenzüberschreitender Verkehr“, „Energie“, „Grenzüberschreitendes Gesundheitswesen" und ,Steuerfragen". (5) Im Aktionsplan fur die deutschfranzösische berufliche Mobilität vom 19.02.2016 hatten beide Bundesministerinnen der Arbeit, Andrea Nahles und Myriam El Khomri, zwölf Maßnahmen zur Förderung der grenzüberschreitenden Mobilität junger Azubis und Arbeitsuchender ergriffen. Dabei werden die Akteure der deutsch-französischen beruflichen Mobilität eingebunden, von den beiden öffentlichen Arbeitsverwaltungen (Bundesagentur für Arbeit und Pôle Emploi) bis zu großen Unternehmen beider Länder (Allianz, BASF, Bosch, Danone, L’Oréal, Michelin).

Ein konkretes Beispiel für grenzüberschreitende Förderung der französischen und deutschen Lernenden findet man in der Oberstufe des beruflichen Gymnasiums (Lycée Professionnel). 2014 wurde für gewerbliche, kaufmännische und hauswirtschaftliche Zweige des beruflichen Gymnasiums das Programm „Azubi-Bacpro“ eingeweiht. Nach dreijähriger dualer Ausbildung und integrierten Praktikumsphasen bekommen der/die deutsche Azubi und der/die französische „Apprenti/e" eine Zusatzqualifikation in der Sprache des Nachbarn. Zurzeit haben acht Gymnasien im Elsass und in Baden-Württemberg diese Partnerschaft vereinbart. Während der Ausbildung dieser Gymnasiasten sind mehrere Austauschphasen und Praktikumsphasen (bis 8 Wochen) im Nachbarland vorgesehen. Das Zertifikat der Zusatzqualifikation wird auf beiden Seiten des Rheins anerkannt und bildet insofern ein großes Plus für die berufliche Karriere der jungen Abiturienten.

Bevor sich eine klare Tendenz in Richtung Stabilisierung, Rückgang oder Anstieg der Deutschlernenden im ganzen Land bestätigt, sind wir auf künftige Zahlen angewiesen, die einen realistischen Vergleich ermöglichen. Im September 2017 wird der ADEAF eine umfangreiche Felduntersuchung zur Entwicklung des Deutschunterrichts in den letzten drei Schuljahren (2015/2016 vor der Reform, 2016/2017 nach der Reform, 2017/2018 nach der Lockerung bzw. Anpassung der 
Deutsch lernen nach (und trotz) der Bildungsreform in Frankreich - eine Zwischenbilanz

L. GAUTHEROT
BIBLIOGRAPHISCHE HINWEISE LITERATURVERZEICHNIS
Reform) in ganz Frankreich einleiten. Auch wenn die Abschaffung der bilingualen Klassen nicht weiter umgesetzt wird, ist deren Wiedereröffnung bei weitem nicht automatisch. Zurzeit hängt der Erhalt eines bilingualen Fremdsprachenunterrichtes an erster Stelle von der Schulleitung und dem Verwaltungsrat ab. Für das kommende Schuljahr (2017/2018) sind die meisten bilingualen Fremdsprachenunterrichtszweige in den Collèges hauptsächlich den Bemühungen der Deutschlehrer/innen (gegebenenfalls der kollektiven Lehrkraft) zu verdanken, die anhand des letzten Juni-Erlasses noch vor der Sommerpause mit den schulischen Instanzen um das Weiterbestehen des DaF-Unterrichts ab der 6. Klasse gekämpft haben. In der Tat kann man die späte Verkündung und vage Kommunikation des neuen Ministeriums über den Unterricht einer zweiten Fremdsprache ab der 6 . Klasse bedauern, die nicht ermöglicht haben, dass sie überall in Frankreich in der allgemeinen Planung und Stundenverteilung der Collèges berücksichtigt und von den Eltern wahrgenommen wurden. Vor allem lassen sich die dramatischen Folgen der Ankündigung der Abschaffung der bilingualen Klassen nicht wiedergutmachen, da wo die Ankündigung manche Eltern davon abgehalten hat, ihre Kinder in einem Zweig (wieder)einzuschreiben, der zum Verschwinden verurteilt war. Es ist also nicht zu bestreiten, dass die Schulreform der vorherigen Bildungsministerin Vallaud-Belkacem obwohl sie nur anderthalb Jahre bestand - katastrophale Auswirkungen auf den Deutschunterricht gehabt hat. Ob der DaFUnterricht trotz Entwarnung und kompensatorischer Maßnahmen zum Aussterben verurteilt wurde, wird erst nach ein paar Jahren festgestellt werden können.

AMMON, Ulrich, 2015. Die Stellung der deutschen Sprache in der Welt. Berlin / München: De Gruyter Mouton.

DURAND, Marie-Laure, 2017 [im Druck]. „L'enseignement de l'allemand et la réforme du collège 2016: l'argumentation anti-crise du ministère de l'Éducation nationale", in: Cahiers d'Études Germaniques $\mathrm{n}^{\circ} 73,185-202$.

GEIGER-JAILLET, Anémone / ROTHACKER, Léa, 2017. „La réforme du collège vue par les principaux: les débuts de sa mise en ocuvre à l'exemple d'une académie", in: Les Langues Modernes, 2/2017, 83-93.

SCHNEIDER-MIZONY, Odile, 2002: „Deutsch als Fremdsprache und Germanistik in Frankreich", in: Jahrbuch fir internationale Germanistik, Jahrgang XXXIV, Heft 1, Berlin [u.A.]: Peter Lang, 19-25.

COIGNARD, Sophie: „Najat Vallaud-Belkacem, l'apprentie sorcière“, in: Le Point, 20.03.2017 [Online]. 
Deutsch lernen nach (und trotz) der Bildungsreform in Frankreich - eine

Zwischenbilanz

L. GAUTHEROT

PRESSEARTIKEL

[ALLE

INTERNETSEITEN

WURDEN AM

15.07.2017 GEPRÜFT]

WEITERE QUELLEN AUDIOAUFNAHMEN

FRANZÖSISCHE DAFHANDBÜCHER

OFFIZIELLE SCHULBEHÖRDEN FRANKREICHS http://www.lepoint.fr/editos-du-point/sophie-coignard/coignardnajat-vallaud-belkacem-l-apprentie-sorciere-20-03-20172113159_2134.php

MEISTER, Martina: „’Wenn wir uns nicht verstehen, ist das ein Risiko'“, Interview mit Susanne Wasum-Rainer, in: Die Welt, 23.04.2015 [Online].

https://www.welt.de/politik/ausland/article140006107/Wenn-wiruns-nicht-verstehen-ist-das-ein-Risiko.html

WITZTHUM, Thomas Sebastian: „Sprachenstreit belastet Beziehung zu Frankreich“, in: Die Welt, 21.04.2015 [Online]

http://www.welt.de/139889058

Goethe Institut Paris, Die Bildungsreform des Collège und ihre Auswirkungen, Informationsabend am 6.05.2015 [Online].

https://www.goethe.de/ins/fr/de/spr/wdl/zuf/brc.html

Blick und Klick, LernBuch 3ème, Paris: Editions Hatier

Fantastisch! 1ère année, 2016, Paris: Editions Maison des Langues.

Fantastisch! zème année, 2017, Paris: Editions Maison des Langues.

Académie de Strasbourg, Collège 2016, Enseignements Pratiques Interdisciplinaires [Online].

https://www.ac-strasbourg.fr/pedagogie/college2016/enseignementspratiques-interdisciplinaires/

Collège des IA-IPR de l'Académie de Strasbourg, Enseignements Pratiques Interdisciplinaires - Académie de Strasbourg, Mai 2016 [Online]. https://www.ac-strasbourg.fr/fileadmin/pedagogie/college2016/epi/DOG_EPI_IPR_2016____pdf

Ministère de l'Éducation Nationale, Arrêté du 19 mai 2015 relatif à l'organisation des enseignements dans les classes de college [Online]

https://www.legifrance.gouv.fr/affichTexte.do?cidTexte=JORFTEXT000030613339

Ministère de l'Éducation Nationale, JORF n ${ }^{\circ} 0142$ du 18 juin 2017, Arrêté du 16 juin 2017 modiffunt l'arrêté du 19 mai 2015 relatif à l'organisation des enseignements dans les classes de college [Online]

https://www.legifrance.gouv.fr/affich Texte.do?cidTexte=JORFTEXT000034952173\&dateTexte $=$ \&categorieLien $=i d$

Ministère de l'Éducation Nationale, Communiqué de presse: „, $+6 \%$ de collégiens germanistes à la rentrée 2016: engagement tenu“, 17.10.2016 [Online]

http://www.education.gouv.fr/cid107855/-6-de-collegiens-germanistes-a-la-rentree-2016-engagement-tenu.html 
Deutsch lernen nach (und trotz) der Bildungsreform in Frankreich - eine Zwischenbilanz

L. GAUTHEROT

\section{OFFIZIELLE DEUT- SCHE BEHÖRDEN}

\section{DEUTSCHLEHRERVE RBAND ADEAF}

Auswärtiges Amt der Bundesrepublik Deutschland. Erklärung von Saarbrücken zur deutsch-französischen Zusammenarbeit in den Grenzregionen, 15.07.2013 [Online]

https://www.saarland.de/dokumente/res_stk/dt-frzGrenzgipfel_Erklaerung_DEU.pdf

Bundesministerium für Arbeit und Soziales / Ministère du travail, de l'emploi de la formation professionnelle et du dialogue social. Aktionsplan für die deutsch-französische berufliche Mobilität, 19.02.2016 [Online].

http://www.bmas.de/SharedDocs/Downloads/DE/PDFMeldungen/2016/aktionsplan.pdf?_ blob=publicationFile\&v=1

„Évolution des effectifs à la rentrée 2016 - Résultats de l'enquête“, 2016 [Online].

http://adeaf.net/spip.php?article138

„Politique des langues et allemand. Contribution de l'ADEAF“, 2017 [Online].

http://adeaf.net/IMG/pdf/politique_des_langues_et_allemand_contribution_de_l_adeaf_2017.pdf

\section{FUßNOTEN}

(1) Über den vermeintlichen ,elitären Charakter“ der bilingualen Klassen lässt sich streiten. In ihrem Vortrag betonte Thérèse Clerc, dass diese Klassen, die „ein hochwertiges, qualitatives und den meistmöglichen Lernenden aller Stadtteile und Herkunft zugänglich sind, gerade das Prinzip der republikanischen Gleichheit verkörpern“ (Informationsabend im Goethe Institut, 6.05.2017 [Online], frei übersetzt nach L.G.), während das Bildungsministerium in seinem öffentlichen Diskurs lieber auf das Wort „Exzellenz" zurückgreift. Witzthum erinnert daran, dass Vallaud-Belkacem selber bei der Aufnahmeprüfung der Verwaltungsschule École Nationale d'Administration zweimal gescheitert ist und liest aus dieser anti-elitären Mission einen persönlichen Nachholbedarf (Revanche-Bedarf?). Siehe den Artikel: „Sprachenstreit belastet Beziehung zu Frankreich“, in: Die Welt, 21.04.2015 [Online].

(2) http://www.education.gouv.fr/cid107855/-6-de-collegiens-germanistes-a-la-rentree-2016-engagement-tenu.html

(3) ,Die EPIs bieten auch die willkommene Gelegenheit an, die erlernten Fremdsprachen und die digitalen Medien in die Praxis umzusetzen" (frei übersetzt nach L.G) in: Enseignements Pratiques Interdisciplinaires Académie de Strasbourg, Mai 2016: 1 [Onlinc].

(4) Eine Ausnahme bilden die LehrerInnen des beruflichen Zweiges der Oberstufe, die gegebenenfalls auch in der entsprechenden 9. Klasse „3ème prépa-pro“ (,,3ème préparatoire à l'enseignement professionnel") unterrichten. Diese bivalenten LehrerInnen unterrichten oft Französisch und Deutsch.

[Online]

(5) Erklärung von Saarbrücken zur deutsch-französischen Zusammenarbeit in den Grenzregionen, 15.07.2013 